\title{
Melhoria do desempenho da Hibridização GA-PSO através do Peso Inercial Adaptativo Fuzzy
}

\author{
Rodrigo Possidônio Noronha ${ }^{1}$ \\ Instituto Federal de Educação, Ciência e Tecnologia do Maranhão, Imperatriz, MA
}

\begin{abstract}
Resumo. A hibridização de algoritmos de otimização pertencentes à teoria da computação evolucionária é realizada com o objetivo de combinar características desejáveis e atenuar características indesejáveis dos métodos envolvidos. De forma específica para uma hibridização envolvendo o $G e$ netic Algorithm (GA) e Particle Swarm Optimization (PSO), embora o GA insira bastante diversidade de posições no espaço de busca permitindo contornar com uma maior facilidade a convergência prematura, o processo de busca converge lentamente. Além disso, somente inserir diversidade de posições não é o suficiente para contornar a convergência prematura em problemas com maior complexidade. Para obter um processo de busca com uma convergência rápida e não prematura, é necessário realizar um bom trade-off entre a busca global e local. Nessa hibridização, embora não seja trivial, o trade-off pode ser realizado, por exemplo, através de uma boa seleção de valores para o peso inercial do PSO. Para isso, nesse artigo, é proposta a utilização de um sistema de inferência fuzzy Mamdani para adaptação paramétrica, a cada iteração, do peso inercial. Sendo assim, é possível melhorar o desempenho da hibridização GA-PSO através de um bom trade-off entre a busca global e local.
\end{abstract}

Palavras-chave. Computação Evolucionária, Genetic Algorithm, Hibridização, Particle Swarm Optimization, Sistema de Inferência Fuzzy Mamdani.

\section{Introdução}

Algoritmos de otimização pertencentes à teoria de computação evolucionária têm sido utilizados para resolver problemas complexos em várias áreas do conhecimento, de tal forma que, além de não necessitar que a função de custo seja contínua no espaço de busca, solucionam com uma maior facilidade problemas multimodais, não lineares, multidimensionais, e entre outros. Dentre a variedade de algoritmos, pode-se citar o GA e PSO, que têm sido amplamente utilizados devido ao robusto e eficiente desempenho na otimização de problemas complexos do mundo real [1]. O PSO, proposto por Kennedy J. e Eberhart R., é um método de otimização da teoria de inteligência de enxames bio-inspirando no comportamento individual e social de animais (pássaros, e entre outros) em bando, por exemplo, em busca de alimentos [2]. O processo de busca realizado pelo PSO converge rapidamente para a solução ótima em problemas unimodais. Porém, em problemas multimodais, devido a ausência de um mecanismo que insira diversidade de posições que torna o processo de busca unidirecional conforme descrito em [2], a convergência prematura pode vir a ocorrer. O GA, proposto por Holland J. H., é um método de otimização bio-inspirado na evolução genética de seres vivos [4]. Através da utilização dos operadores genéticos, a cada geração ou iteração, uma grande diversidade de posições é inserida no espaço de busca. Devido a isso, naturalmente, o GA apresenta uma maior facilidade em contornar a convergência prematura em

\footnotetext{
${ }^{1}$ rodrigo.noronha@ifma.edu.br
} 
problemas multimodais, quando comparado ao PSO; porém, devido a grande diversidade inserida que torna o processo de busca multidirecional, a convergência do GA é lenta [3]. A convergência prematura do processo de busca realizado pelo PSO e a lenta convergência do processo de busca realizado pelo GA são resultados de um ineficiente trade-off entre a busca global e local.

A partir da década de 1980, a hibridização de algoritmos de otimização pertencentes à teoria de computação evolucionária tem sido recorrente, uma vez que é possível combinar as características desejáveis e atenuar as características indesejáveis dos algoritmos envolvidos. Originalmente, a hibridização GA-PSO é realizada com o objetivo de obter um algoritmo de otimização que tenha a capacidade de otimizar problemas multimodais com uma convergência rápida e com grande diversidade. Existem algumas contribuições à hibridização GA-PSO publicadas na literatura, como em [5] que a proposta é baseada em codificação paralela bidimensional com o objetivo de otimizar os recursos para bloqueio de Jammers. Em [8], a hibridização GA-PSO foi proposta para com o objetivo de diagnosticar falhas em rolamentos mecânicos. Em [6], os parâmetros de controladores fuzzy tipo 1 e tipo 2 foram sintonizados pela hibridização GA-PSO. É importante notar que devido a grande diversidade de posições no espaço de busca inserida pelo GA, o processo de busca realizado pela hibridização converge lentamente. Além disso, embora o processo de busca realizado hibridização tenha uma maior facilidade em contornar a convergência prematura, apenas inserir diversidade no espaço de busca pode não ser o suficiente. Em função da complexidade do problema (por exemplo, quantidade de modos, dimensão do espaço de busca, não linearidade, e entre outros), a convergência prematura pode ocorrer devido ao excesso ou insuficiência de diversidade. Uma vez que a diversidade é uma medida de distância entre as partículas no espaço de busca, através de um bom trade-off entre a busca global e local é possível evitar a convergência prematura e, além disso, realizar um processo de busca que convirja rapidamente. Na hibridização GA-PSO, embora não seja trivial, um bom trade-off entre a busca global e local pode ser realizado, por exemplo, através de uma boa seleção de valores para o peso inercial [7].

Neste artigo, é proposto a utilização de um sistema de inferência fuzzy Mamdani (do inglês, MFIS) visando, a cada iteração, realizar a adaptação paramétrica do peso inercial. A justificativa para descrever o comportamento dinâmico do peso inercial em um contexto fuzzy, é devido a capacidade de um MFIS representar o conhecimento subjetivo em uma base de regras; dessa forma, o comportamento dinâmico do peso inercial é descrito em função do conhecimento do especialista sobre como deve ser realizado o trade-off. Portanto, essa é a motivação para essa proposta, que é clara e justificada. As entradas do MFIS são a diversidade e a iteração normalizadas pelo método Min-Max; a saída é o peso inercial. Dessa forma, o peso inercial é adaptado parametricamente em função de informações sobre a distância entre as partículas e sobre o avanço temporal do processo de busca. Esse artigo é organizado da seguinte forma: na Seção 2 são apresentadas as declarações do problema que a metodologia de otimização proposta vista solucionar; na Seção 3, é apresentada a metodologia de otimização proposta, que é organizada em cinco subseções; na Seção 4, são apresentados os resultados computacionais obtidos através da otimização de benchmarks.

\section{Declarações do Problema}

O objetivo da metodologia de otimização proposta é realizar a busca por soluções que maximize ou minimize uma função de custo, formulado como:

$$
\left\{\begin{array}{l}
J=J\left(x_{i, 1}[k], x_{i, 2}[k], \ldots, x_{i, n}[k]\right) \\
\text { sujeito } a: \\
\min \left(\mathbf{x}_{i}\right) \leq \mathbf{x}_{i}[k] \leq \max \left(\mathbf{x}_{i}\right) \text { e } \min \left(\mathbf{v}_{i}\right) \leq \mathbf{v}_{i}[k] \leq \max \left(\mathbf{v}_{i}\right) \\
k \in[1, K]
\end{array}\right.
$$


onde, $J: \mathbb{R}^{n} \rightarrow \mathbb{R}$ é a função de custo, $\mathbf{v}_{i}[k]=\left[v_{i, 1}[k], v_{i, 2}[k], \ldots, v_{i, n}[k]\right] \in \mathbb{R}^{1 \times n}$ e $\mathbf{x}_{i}[k]=$ $\left[x_{i, 1}[k], x_{i, 2}[k], \ldots, x_{i, n}[k]\right] \in \mathbb{R}^{1 \times n}$ são, respectivamente, os vetores de velocidade e posição da $i$ ésima partícula pertencente a uma população de $N$ partículas. Para um problema de minimização, a atualização do vetor de melhor posição individual $\mathbf{p}_{i}[k]$ da $i$-ésima partícula, é dado por:

$$
\mathbf{p}_{i}[k+1]=\left\{\begin{array}{l}
\mathbf{p}_{i}[k] \text { se } J\left(\mathbf{x}_{i}[k+1]\right) \geq J\left(\mathbf{p}_{i}[k]\right) \\
\mathbf{x}_{i}[k+1] \text { se } J\left(\mathbf{x}_{i}[k+1]\right)<J\left(\mathbf{p}_{i}[k]\right)
\end{array}\right.
$$

para um problema de maximização, a atualização é dada por:

$$
\mathbf{p}_{i}[k+1]=\left\{\begin{array}{l}
\mathbf{p}_{i}[k] \text { se } J\left(\mathbf{x}_{i}[k+1] \leq J\left(\mathbf{p}_{i}[k]\right)\right. \\
\mathbf{x}_{i}[k+1] \text { se } J\left(\mathbf{x}_{i}[k+1]\right)>J\left(\mathbf{p}_{i}[k]\right)
\end{array}\right.
$$

Para um problema de minimização, a atualização do vetor de melhor posição global $\mathbf{p}_{g}[k]$, é dado por:

$$
\mathbf{p}_{g}[k+1]=\arg \min \left\{\mathbf{p}_{1}[k+1], \mathbf{p}_{2}[k+1], \ldots, \mathbf{p}_{N}[k+1]\right\}
$$

para um problema de maximização, a atualização é dada por:

$$
\mathbf{p}_{g}[k+1]=\arg \max \left\{\mathbf{p}_{1}[k+1], \mathbf{p}_{2}[k+1], \ldots, \mathbf{p}_{N}[k+1]\right\}
$$

\section{Metodologia de Otimização Proposta}

Nesta seção, é realizada a descrição da metodologia de otimização proposta. A cada iteração $k$, o vetor posição $\mathbf{x}_{i}[k]$ é atualizado pelos operadores genéticos de seleção de pais, crossover e mutação. Logo após, os novos vetores posição são avaliados pela função de custo e são atualizadas os vetores das melhores posições individual $\mathbf{p}_{i}[k]$ e o vetor da melhor posição global $\mathbf{p}_{g}[k]$. Logo em seguida, são calculadas a diversidade normalizada e a normalização da iteração pelo método Min-Max. Após feito isso, é executado o MFIS, no qual a saída é o peso inercial adaptado. Após realizada a adaptação do peso inercial, são atualizados os vetores de velocidade e posição, $\mathbf{v}_{i}[k]$ e $\mathbf{x}_{i}[k]$. Por fim, são atualizados os vetores de melhor posição individual $\mathbf{p}_{i}[k]$ e de melhor posição global $\mathbf{p}_{g}[k]$. Se a condição de parada for satisfeita, então é obtida a solução para o problema; caso contrário, a busca pela solução ótima é continuada.

\subsection{Operador Genético de Seleção de Pais}

Através do operador genético de seleção de pais, é possível selecionar as partículas com melhor custo, para serem reproduzidas pelo operador genético crossover, de acordo com a taxa de crossover $\mu_{c}$. O método de seleção de pais utilizado nessa metodologia é do tipo torneio, que seleciona, aleatoriamente, $m$ partículas da população para participarem do torneio, no qual a partícula com melhor custo será a partícula pai da próxima geração, conforme a pseudocódigo a seguir:

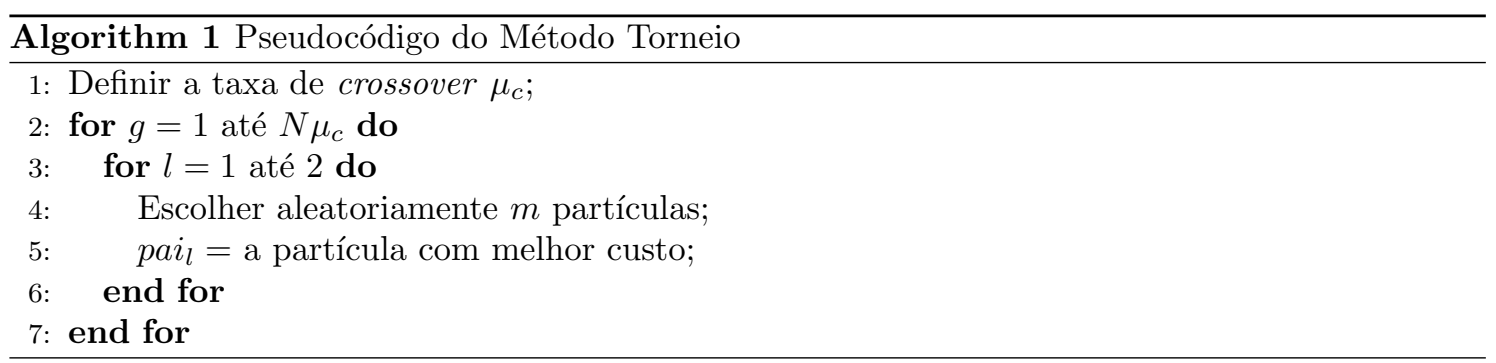




\subsection{Operador Genético de Crossover}

Após realizada a seleção das partículas com melhores custos, é realizado o crossover. O operador genético de crossover é utilizado para combinar as características dos pais selecionados, visando obter partículas mais aptas a serem possíveis soluções para o problema. Valores típicos de $\mu_{c}$ estão no intervalo $[0,51]$. O método de crossover utilizado nessa metodologia é do tipo uniforme, conforme o pseudocódigo a seguir:

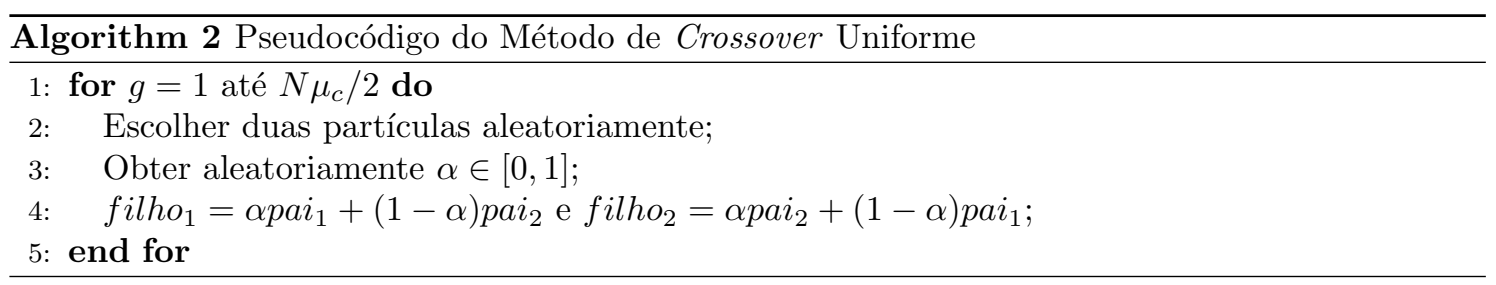

\subsection{Operador Genético de Mutação}

O próximo passo após a realização do crossover é realizar a mutação dos genes das partículas de uma população. Através do operador genético de mutação, é possível explorar novas regiões no espaço de busca. A mutação modifica, com uma taxa $\mu_{m}$, alguma característica da partícula. Sendo assim, o uso do operador genético de mutação é importante para inserir diversidade de posições no espaço de busca. Valores típicos de $\mu_{m}$ estão no intervalo [0,005 0,05]. O método de mutação utilizado nessa metodologia é do tipo aleatório, conforme pseudocódigo a seguir:

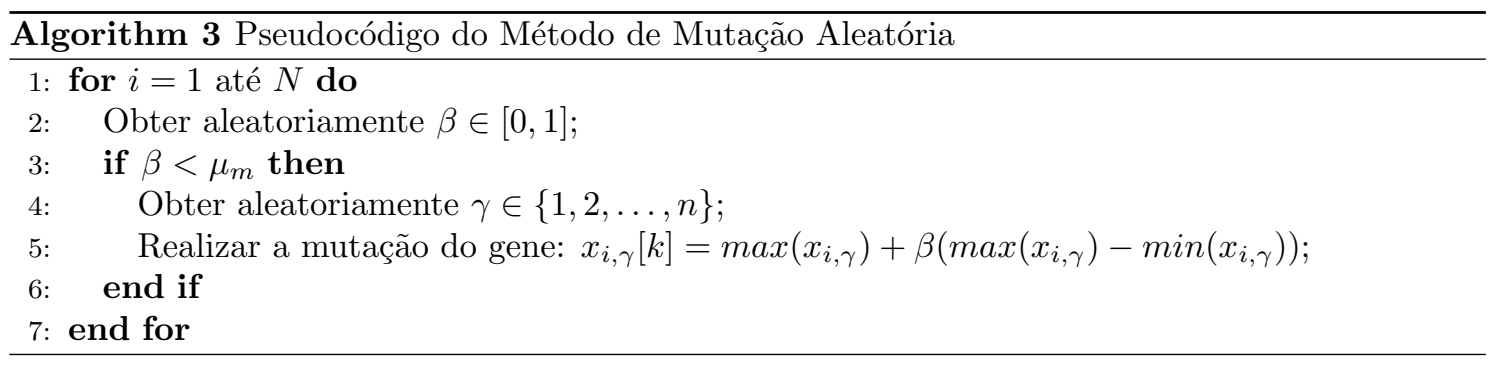

\subsection{Peso Inercial Adaptativo Fuzzy}

Após executados os operadores genéticos,, o próximo passo é a atualização do peso inercial através de um MFIS. A adaptação do peso inercial é descrita a seguir:

$$
\begin{gathered}
\omega[k]=\operatorname{MFIS}(\mathcal{D}[k], \mathcal{K}[k]) \\
\mathcal{D}[k]=\frac{D[k]-d_{\text {min }}[k]}{d_{\text {max }}[k]-d_{\text {min }}[k]} \text { e } \mathcal{K}[k]=\frac{k-1}{K-1}
\end{gathered}
$$

em que, $D[k]$ é a medida de diversidade dada pela distância Euclidiana média entre o vetor de posição $x_{i}[k]$ e o vetor de melhor posição global $p_{g}[k]$. As variáveis $d_{\min }[k]$ e $d_{\text {max }}[k]$ são, respectivamente, o menor e maior valor obtidos para diversidade até a iteração $k$. As variáveis $\mathcal{D}[k]$ e $\mathcal{K}[k]$ são, respectivamente, a diversidade e iteração normalizadas pelo método Min-Max. As entradas do MFIS, que são variáveis linguísticas, são a diversidade normalizada e a iteração normalizada. A cada iteração $k$, através da fuzzificação, a $j$-ésima função de pertinência (do inglês, MBF) realiza o mapeamento dos universos de discurso das variáveis linguísticas de entrada do MFIS para o intervalo $[0,1]$ de grau de pertinência, ou seja, $m_{j}(\mathcal{K}[k]): \mathbb{R} \rightarrow[0,1]$ e $m_{j}(\mathcal{D}[k]): \mathbb{R} \rightarrow[0,1]$, para 
$j=1,2,3$. As MBFs são definidas em função do conhecimento do especialista sobre como deve ser realizado o trade-off entre a busca global e local. Para cada variável linguística de entrada do MFIS, foram definidas três MBFs do tipo triangular, com os valores linguísticos "pequeno" $(P)$, "médio" $(M)$ e "grande" $(G)$, cujo os parâmetros podem ser vistos na Tabela 1. As variáveis linguísticas são associadas aos valores linguísticos por meio de proposições fuzzy, que são conectadas através de conectivos lógicos no antecedente e consequente, formando regras fuzzy conforme o seguinte exemplo $\mathcal{R}^{i}$ : Se $\mathcal{K}$ é P e $\mathcal{D}$ é P então $\omega^{i}$ é G. Na base de regras fuzzy desenvolvida, conforme pode ser visto em (7), as proposições fuzzy do antecedente são conectadas por conectivos lógicos "e", que realiza a seguinte operação: $t\left[m_{j}(\mathcal{K}[k]), m_{j}(\mathcal{D}[k])\right]=\min \left(m_{j}(\mathcal{K}[k]), m_{j}(\mathcal{D}[k])\right)$, em que $t[\bullet]$ representa a norma-t. Após o cálculo da norma-t, é obtido o grau de pertinência de ativação da $i$-ésima regra fuzzy, que seleciona o valor mínimo entre dois graus de pertinência.

$$
\begin{aligned}
& \mathcal{R}^{1}: \text { Se } \mathcal{K} \text { é } \mathrm{P} \text { e } \mathcal{D} \text { é } \mathrm{P} \text { então } \omega^{1} \text { é } \mathrm{G} \\
& \mathcal{R}^{3} \text { : Se } \mathcal{K} \text { é } \mathrm{P} \text { e } \mathcal{D} \text { é } \mathrm{G} \text { então } \omega^{5} \text { é } \mathrm{P} \\
& \mathcal{R}^{2}: \text { Se } \mathcal{K} \text { é } \mathrm{P} \text { e } \mathcal{D} \text { é } \mathrm{M} \text { então } \omega^{2} \text { é } \mathrm{M} \\
& \mathcal{R}^{4}: \text { Se } \mathcal{K} \text { é } \mathrm{M} \text { e } \mathcal{D} \text { é } \mathrm{P} \text { então } \omega^{4} \text { é } \mathrm{M} \\
& \mathcal{R}^{5}: \text { Se } \mathcal{K} \text { é } \mathrm{M} \text { e } \mathcal{D} \text { é } \mathrm{M} \text { então } \omega^{5} \text { é } \mathrm{M} \\
& \mathcal{R}^{6}: \text { Se } \mathcal{K} \text { é } \mathrm{M} \text { e } \mathcal{D} \text { é } \mathrm{G} \text { então } \omega^{6} \text { é } \mathrm{M} \\
& \mathcal{R}^{7} \text { : Se } \mathcal{K} \text { é } \mathrm{G} \text { e } \mathcal{D} \text { é } \mathrm{P} \text { então } \omega^{7} \text { é } \mathrm{M} \\
& \mathcal{R}^{8} \text { : Se } \mathcal{K} \text { é } \mathrm{G} \text { e } \mathcal{D} \text { é } \mathrm{M} \text { então } \omega^{8} \text { é } \mathrm{P}
\end{aligned}
$$

Após obtido o grau de pertinência de ativação da $i$-ésima regra fuzzy, em seguida é calculada a implicação fuzzy do consequente. A entrada da implicação fuzzy é o grau de pertinência de ativação da $i$-ésima regra fuzzy, e a saída é uma MBF obtido através do operador mínimo (min). Cada regra fuzzy é ativada com um determinado grau de pertinência, sendo assim, as MBFs de saída precisam ser combinados para que o MFIS possa ter uma resposta total. Através da agregação, todas as MBFs de saída são combinados para se obter uma única MBF. Após realizada a agregação, é necessário obter um valor numérico (crisp) de saída. Para isso, é realizada a defuzzificação do MBF de saída obtido pela agregação. Nessa metodologia, o método de defuzzificação utilizado é do tipo centroide, conforme a seguir: $\omega[k]=\sum_{i=1}^{9} \omega^{i}[k] m_{j}\left(\omega^{i}[k]\right)\left(\sum_{i=1}^{9} m_{j}\left(\omega^{i}[k]\right)\right)^{-1}$ no qual, para a variável linguística de saída do MFIS, foram definidas três MBFs do tipo triangular, com os valores linguísticos "pequeno" $(\mathrm{P})$, "médio $(\mathrm{M})$ e "grande" $(\mathrm{G})$, cujo os parâmetros podem ser vistos na Tabela 1.

Tabela 1: Intervalos paramétricos das MBFs triangulares do antecedente e consequente.

\begin{tabular}{c|c|c|c|c|c}
\hline \hline \multicolumn{2}{c|}{$\mathcal{K}[k]$} & \multicolumn{2}{c|}{$\mathcal{D}[k]$} & \multicolumn{2}{c}{$\omega^{i}[k]$} \\
\hline Valor Linguístico & Intervalo & Valor Linguístico & Intervalo & Valor Linguístico & Intervalo \\
\hline Pequeno & {$\left[\begin{array}{lll}0 & 0 & 0,1\end{array}\right]$} & Pequeno & {$\left[\begin{array}{lll}0 & 0,5\end{array}\right]$} & Pequeno & {$\left[\begin{array}{lll}0,001 & 0,09 & 0,15\end{array}\right]$} \\
\hline Médio & {$\left[\begin{array}{lll}0 & 0,1 & 0,3\end{array}\right]$} & Médio & {$\left[\begin{array}{lll}0 & 0,5 & 1\end{array}\right]$} & Médio & {$\left[\begin{array}{lll}0,09 & 0,5 & 0,35\end{array}\right]$} \\
\hline Grande & {$\left[\begin{array}{llll}0,1 & 0,3 & 1,0\end{array}\right]$} & Grande & {$\left[\begin{array}{lll}0,5 & 1,0 & 1,0\end{array}\right]$} & Grande & {$\left[\begin{array}{lll}0,15 & 0,35 & 0,9\end{array}\right]$} \\
\hline \hline
\end{tabular}

\subsection{Atualização dos Vetores de Velocidade e Posição das Partículas}

Após a adaptação fuzzy do peso inercial, o próximo passo é a atualização dos vetores de velocidade e posição. O vetor velocidade $\mathbf{v}_{i}[k]$ tem a função de controlar a atualização do vetor de posição $\mathbf{x}_{i}[k]$. A equação de atualização do vetor velocidade é composta por dois termos que introduzem a natureza estocástica ao processo de busca $r_{1}, r_{2} \in[0,1]$, dos coeficientes de aceleração $C_{1}, C_{2} \in[0,2]$ e do peso inercial $\omega \in[0,1,2]$. As equações de atualização do vetor velocidade e posição da $i$-ésima partícula, são dadas, respectivamente, por:

$$
\begin{gathered}
v_{i, j}[k+1]=v_{i, j}[k] \omega+C_{1} r_{1}\left(p_{i, j}[k]-x_{i, j}[k]\right)+C_{2} r_{2}\left(p_{g, j}[k]-x_{i, j}[k]\right) \\
\mathbf{x}_{i}[k+1]=\mathbf{x}_{i}[k]+\mathbf{v}_{i}[k+1]
\end{gathered}
$$




\section{Resultados Computacionais}

Para validar a metodologia de otimização proposta, nessa seção é realizada a minimização de dois benchmarks, que são a função Rastrigin $\left(f_{1}[\mathbf{x}]\right)$ e função Griewank $\left(f_{2}[\mathbf{x}]\right)$, descritas a seguir:

$$
f_{1}(\mathbf{x}[k])=10 K+\sum_{k=1}^{K}\left(\mathbf{x}[k]^{2}-10 \cos 2 \pi \mathbf{x}[k]\right) \quad f_{2}(\mathbf{x}[k])=1+\frac{1}{4000} \sum_{k=1}^{K} \mathbf{x}[k]^{2}-\prod_{k=1}^{K} \cos \frac{\mathbf{x}[k]}{\sqrt{k}}
$$

Os resultados apresentados na Tabela 2, foram obtidos pela definição dos seguintes parâmetros: taxa de crossover $\mu_{c}=0,9$, taxa de mutação $\mu_{m}=0,1$, coeficiente de aceleração $C_{1}=C_{2}=2$ e o peso inercial $\omega=1$ (para o PSO tradicional). O parâmetro $m$ utilizado para selecionar as partículas pais pelo método do torneio foi definido como $m=3$. Os vetores de posição inicial $\mathbf{x}_{i}[1]$ foram inicializados de forma aleatória e sendo iguais para todos os algoritmos. Os vetores de velocidade e de melhores posições individuais foram inicializados como $\mathbf{p}_{i}[1]=\mathbf{x}_{i}[1]$ e $\mathbf{v}_{i}[1]=\mathbf{0}$. O tamanho da população foi definida como $N=100$ partículas e foram avaliadas durante $K=300$ iterações. Uma vez que todos os algoritmos avaliados na Tabela 2 são estocásticos, então, o processo de busca, para cada algoritmo, foi realizado 100 vezes com o objetivo de obter uma maior coerência estatística dos resultados obtidos. A dimensão do espaço de busca foi definida como $n=20$. Devido aos algoritmos terem desenvolvido os piores resultados na otimização do benchmark $f_{1}(\mathbf{x}[k])$, assim a análise do desempenho será realizada apenas nesse benchmark; é importante notar que o mínimo global do benchmark $f_{1}(\mathbf{x}[k])$ é igual a zero e o espaço de busca é limitado ao intervalo [-5, 10].

Tabela 2: Resultados obtidos para 100 realizações do processo de busca.

\begin{tabular}{c|c|c|c}
\hline \hline Algoritmo & Resultados & $f_{1}(\mathbf{x}[k])$ & $f_{2}(\mathbf{x}[k])$ \\
\hline \multirow{2}{*}{ PSO } & Custo Médio & 163,0306 & 0,0017 \\
\cline { 2 - 4 } & Desvio Padrão & 162,1489 & 0,0028 \\
\hline \hline \multirow{2}{*}{ GA } & Custo Médio & 8,0351 & 0,0069 \\
\cline { 2 - 4 } & Desvio Padrão & 8,1492 & 0,0032 \\
\hline \hline \multirow{2}{*}{ GA-PSO } & Custo Médio & 3,9898 & 0 \\
\cline { 2 - 4 } & Desvio Padrão & 4,9871 & 0 \\
\hline \hline \multirow{2}{*}{ Proposto } & Custo Médio & 0,5912 & 0 \\
\cline { 2 - 4 } & Desvio Padrão & 0,5601 & 0 \\
\hline \hline
\end{tabular}

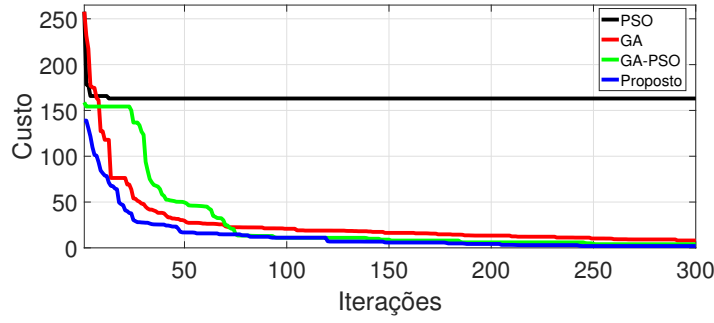

Figura 1: Custo para o benchmark $f_{1}(\mathbf{x}[k])$.

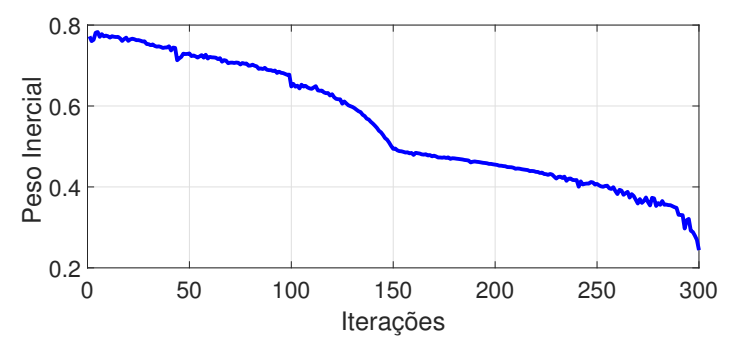

Figura 2: Peso inercial do benchmark $f_{1}(\mathbf{x}[k])$.

Através da Figura 1, nota-se que o PSO convergiu prematuramente, uma vez que o benchmark $f_{1}(\mathbf{x}[k])$ é multimodal; esse desempenho insatisfatório pode ser confirmado através da Tabela 2. Além do PSO, na Figura 1 são mostrados os resultados obtidos pelo algoritmo GA-PSO (sem a adaptação fuzzy do peso inercial) e pelo GA. Nota-se que o GA convergiu mais lentamente do que o GA-PSO, que é devido ao processo de busca ser realizado unicamente de forma multidirecional; o 
GA-PSO obteve uma convergência mais rápida do que o GA, que é devido ao processo de busca ser realizado tanto de forma multidirecional como unidirecional. Já em relação ao algoritmo proposto, que é o GA-PSO com o peso inercial adaptativo fuzzy, nota-se que, além de evitar a convergência prematura, o algoritmo obteve a convergência mais rápida e os melhores resultados de otimização, de acordo com a Tabela 2. A dinâmica de adaptação descrita por um MFIS, permitiu que o peso inercial, durante as primeiras iterações, visando uma busca global, tivesse um valor elevado; ao fim das iterações, visando uma busca local e a convergência, o seu valor foi decrescido, de acordo com a Figura 2. Dessa forma, foi possível obter um bom trade-off entre a busca global e local.

\section{Conclusão}

Nesse artigo, foi proposta um nova versão da hibridização GA-PSO com o peso inercial adaptativo fuzzy, visando uma melhoria no desempenho do algoritmo através de um bom trade-off entre a busca global e local. Nota-se que a adaptação paramétrica realizada pelo MFIS realizou o decrescimento do valor do peso inercial, indo desde uma busca global e, consequentemente realizando uma exploração do espaço de busca, até uma busca local e, consequentemente realizando a convergência. Porém, o decrescimento não foi realizado de forma linear, que é um resultado coerente, uma vez que o processo de busca, que é estocástico, não permite que as partículas desenvolvam uma comportamento linear. O comportamento não linear do valor do peso inercial permitiu que, em função de informações sobre as distâncias entre as partículas e do avanço temporal do processo de busca, quando presas em ótimos locais, as partículas saltassem para melhores posições; assim evitando uma convergência prematura e desenvolvendo uma rápida convergência.

\section{Referências}

[1] Ding, Y., Zhang, W., Yu, L., Lu, K. The accuracy and efficiency of GA and PSO optimization schemes on estimating reaction kinetic parameters of biomass pyrolysis, Energy, 176:582-588, 2019. DOI: $10.1016 /$ j.energy.2019.04.030.

[2] Eberhart, R. C., Kennedy, J. Particle swarm optimization, Proceedings of the IEEE international conference on neural networks, 1995. DOI: 10.1109/MHS.1995.494215.

[3] Gao, W. An improved fast-convergent genetic algorithm, EEE International Conference on Robotics, Intelligent Systems and Signal Processing, 2003. DOI: 10.1109/RISSP.2003.1285761.

[4] Holland, J. H. Adaptation in Natural and Artificial Systems. University of Michigan Press, Ann Arbor, 1975.

[5] Liu, F., Wang, Y., Chen, J., Wang, Q., Yuan, N. Research on Jamming Resource Allocation Technology Based on Improved GAPSO Algorithm, Journal of Physics: Conference Series, 1738:012075, 2021.

[6] Martínez-Soto, R., Castillo, O., Aguilar, L. T., Rodriguez, A. A hybrid optimization method with PSO and GA to automatically design Type-1 and Type-2 fuzzy logic controllers, International Journal of Machine Learning and Cybernetics, 6:175-196, 2013.

[7] Shi, Y., Eberhart, R. C. Parameter selection in particle swarm optimization, International Conference on Evolutionary Programming, 591 - 600, 1998. DOI: 10.1007/BFb0040810.

[8] Zhang, X., Zhang, W., Guo, Q., Lei, W. Optimization of HMM Based on Adaptive GAPSO and Its Application in Fault Diagnosis of Rolling Bearing, 5th International Conference on Control and Robotics Engineering (ICCRE), 53-57, 2020. 